Supporting information

\title{
Nanostructuration of PEDOT in Porous Coordination Polymers for Tunable Porosity and Conductivity
}

\author{
Benjamin Le Ouay, ${ }^{\dagger},{ }^{\dagger}$ Mickael Boudot, ${ }^{\S}$ Takashi Kitao, ${ }^{\dagger}$ Takeshi Yanagida, ${ }^{\S}$ Susumu \\ Kitagawa, $^{\dagger, \# *}$ and Takashi Uemura ${ }^{\dagger, \ddagger *}$ \\ Department of Synthetic Chemistry and Biological Chemistry, Graduate School of Engineering, Kyoto \\ University, Katsura, Nishikyo-ku, Kyoto 615-8510, Japan \\ ${ }^{\ddagger}$ CREST, Japan Science and Technology Agency (JST), 4-1-8 Honcho, Kawaguchi, Saitama 332-0012, Japan \\ ${ }^{\S}$ Institute for Materials Chemistry and Engineering, Kyushu University, 6-1 Kasuga-Koen, Kasuga, Fukuoka \\ 816-8580, Japan \\ \#Institute for Integrated Cell-Material Sciences (WPI-iCeMS), Kyoto University, Yoshida, Sakyo-ku, Kyoto \\ 606-8501, Japan \\ *uemura@ sbchem.kyoto-u.ac.jp, kitagawa@icems.kyoto-u.ac.jp
}

\section{$\underline{\text { Experimental section }}$}

$\underline{\text { Synthesis of MIL-101 }\left(\mathrm{Cr}_{3}(\mathrm{BDC})_{3} \mathrm{FO}\left(\mathrm{H}_{2} \mathrm{O}_{2}, \mathbf{1}\right)\right.}$

$\mathbf{1}$ was prepared using a scaled-up version of a reported procedure. ${ }^{1} 1,4$-Benzenedicarboxylic acid $\left(\mathrm{H}_{2} \mathrm{BDC}, 8.0 \mathrm{mmol}\right), \mathrm{Cr}\left(\mathrm{NO}_{3}\right)_{3} \cdot 9 \mathrm{H}_{2} \mathrm{O}(8.0 \mathrm{mmol})$, deionized water $(50 \mathrm{~mL})$, and $\mathrm{HF}(48$ $\mathrm{wt} \%$ solution in water, $8.0 \mathrm{mmol} \mathrm{HF}$ ) were introduced in a $100 \mathrm{~mL}$ Teflon-lined autoclave and heated at $220{ }^{\circ} \mathrm{C}$ for $8 \mathrm{~h}$. After cooling, the resulting green powder was filtered over a $100 \mu \mathrm{m}$ grid to remove the unreacted crystals of terephthalic acid, and then isolated using a $0.2 \mu \mathrm{m}$ PTFE membrane. The compound was then purified using solvothermal treatment in EtOH $\left(3 \mathrm{~h}, 80^{\circ} \mathrm{C}\right)$, DMF $\left(3 \mathrm{~h}, 100^{\circ} \mathrm{C}\right)$, and $\mathrm{NH}_{4} \mathrm{~F}\left(1 \mathrm{M}\right.$ in water, $\left.16 \mathrm{~h}, 90^{\circ} \mathrm{C}\right)$. Samples were then washed copiously with water and left to degas under vacuum overnight at $150{ }^{\circ} \mathrm{C}$.

Synthesis of La(BTB) (2)

2 was prepared following an adapted procedure. ${ }^{2}$ 1,3,5-Benzenetrisbenzoic acid $\left(\mathrm{H}_{3} \mathrm{BTB}\right)$ was purchased from TCI, Japan, and other reagents were purchased from Wako Chemicals, Japan. $\mathrm{H}_{3} \mathrm{BTB}(1.00 \mathrm{~g}, 2.27 \mathrm{mmol})$ and $\mathrm{LaCl}_{3} \cdot 7 \mathrm{H}_{2} \mathrm{O}(0.843 \mathrm{~g}, 2.27 \mathrm{mmol})$ were dissolved in an aqueous $\mathrm{NaOH}$ solution $(0.15 \mathrm{~mol} / \mathrm{L}, 25 \mathrm{~mL})$, in a round bottom flask. After 2 min of stirring, hot $\left(40{ }^{\circ} \mathrm{C}\right)$ cyclohexanol $(25 \mathrm{~mL})$ was added. Reaction in the diphasic system was carried out for $24 \mathrm{~h}$ under reflux and vigorous stirring. After the reaction, crystals were collected by filtration over a PTFE membrane filter $(0.2 \mu \mathrm{m}$ pore size, Advantec, Japan $)$ and washed thoroughly with ethanol, water, and acetone, then dried under reduced pressure at 
$150{ }^{\circ} \mathrm{C}$.

Synthesis of PEDOT@PCP composites

1 or 2 (ca. $200 \mathrm{mg}$ ) was degassed in a Schlenk vial overnight at $150{ }^{\circ} \mathrm{C}$. The desired amount of EDOT (3,4-ethylenedioxythiophene) dissolved in diethyl ether (4 mL) was introduced in the Schlenk vial and gently stirred to homogenize the suspension of PCP. Diethyl ether was then removed very carefully by evacuation under reduced pressure (a progressive decrease down to $0.3 \mathrm{kPa}$, then $0.3 \mathrm{kPa}$ for $1 \mathrm{~h}$ ). $\mathrm{I}_{2}$ (2.5 equivalents/EDOT) was then introduced in the Schlenk vial. The content of the Schlenk vial was then placed under reduced pressure $(0.3$ $\mathrm{kPa}$ ) before sealing, and left to react at $90{ }^{\circ} \mathrm{C}$ for $48 \mathrm{~h}$. The reaction could be monitored by the color change, from green to black. Composite powder was then rinsed copiously with methanol, acetone, water, and then acetone until a clear filtrate was obtained, before degassing overnight at $150{ }^{\circ} \mathrm{C}$. Even if a significant fraction of unreacted $\mathrm{I}_{2}$ is removed upon rinsing, some more is evacuated during the degassing step, and adequate measures to trap it should be taken.

Synthesis of PEDOT in solution

For comparison purpose, non-porous PEDOT was prepared by polymerization in solution. $\mathrm{I}_{2}$ $(2.23 \mathrm{~g}, 8.8 \mathrm{mmol})$ was dissolved in $25 \mathrm{~mL}$ toluene. EDOT $(0.50 \mathrm{~g}, 3.5 \mathrm{mmol})$ was then added and polymerization was carried out at $90{ }^{\circ} \mathrm{C}$ for $16 \mathrm{~h}$. The black precipitate was then collected by filtration, rinsed with acetone, and degassed overnight at $150{ }^{\circ} \mathrm{C}$ under reduced pressure.

\section{Procedure for PEDOT isolation}

PEDOT was extracted by successively immersing the composite powder for $2 \mathrm{~h}$ in an ethanolic hydrazine solution (5 vol.\% hydrazine monohydrate), then for $16 \mathrm{~h}$ in an aqueous $\mathrm{NaOH}$ solution $\left(2 \mathrm{~mol} . \mathrm{L}^{-1}\right)$. The resulting dedoped PEDOT was then rinsed thoroughly with water, and dried under vacuum at $150{ }^{\circ} \mathrm{C}$.

\section{Instrumental section}

Powder X-ray diffractometry

PXRD diagrams were collected on a Rigaku SmartLab Diffractometer, using a $\mathrm{Cu}$ anode ( $\lambda$ $=0.154 \mathrm{~nm})$.

$\underline{\mathrm{N}_{2}}$ adsorption measurement

$\mathrm{N}_{2}$ adsorption measurements were performed on a Belsorp II mini (BEL-Japan, Inc.). Samples were activated at $150{ }^{\circ} \mathrm{C}$ for $16 \mathrm{~h}$ before the measurement. 


\section{$\underline{X-r a y ~ f l u o r e s c e n c e ~}$}

The X-ray fluorescence spectroscopy was performed using a Rigaku EDXL300 spectrometer. Sample analysis was performed on pressure sintered pellets (5 mm, $10 \mathrm{MPa})$.

Thermo-gravimetric analysis

TGA plots were recorded using a Rigaku TG 8120. Samples were heated in an aluminum pan $\left(500{ }^{\circ} \mathrm{C}, 10^{\circ} \mathrm{C} / \mathrm{min}\right)$ under a $\mathrm{N}_{2}$ flux $(60 \mathrm{~mL} / \mathrm{min})$.

Infrared spectroscopy

Attenuated total reflection-infrared spectra were collected on a JASCO FT/IR 4200 spectrometer.

\section{Raman spectroscopy}

Raman spectra were collected on a HORIBA LabRAM HR-Evolution spectrometer, using a laser source (wavelength: $532 \mathrm{~nm}$ ) for excitation.

\section{MALDI-TOF}

Matrix-assisted laser desorption/ionization time-of-flight mass spectrometry (MALDI-TOF MS) spectra were recorded on an ultraflex instrument (Bruker Daltonics) using TTh as the matrix.

\section{Gel permeation chromatography}

Gel permeation chromatography (GPC) measurements on the resulting polymers were performed in DMF at $40{ }^{\circ} \mathrm{C}$ on three linear-type polystyrene gel columns (Shodex K-805L) that were connected to a JASCO PU-980 precision pump, a JASCO RI-930 refractive index detector, and a JASCO UV-970 UV/vis detector set at $256 \mathrm{~nm}$. The columns were calibrated against standard polystyrene samples.

\section{Impedance spectroscopy measurement}

Samples (ca. $30 \mathrm{mg}$ ) were finely ground in a mortar, then pressure-sintered (10 MPa) to form pellets ( $5 \mathrm{~mm}$ diameter, ca. $1 \mathrm{~mm}$ thickness). Measurements (two-electrodes set-up) were performed using a Solartron SI 1260 phase analyzer coupled to a Solartron SI 1296 dielectric interface.

\section{$\mathrm{NO}_{2}$ sensing experiment}

Platinum interdigitated electrodes (concentric design) on a glass substrate were obtained 
from DropSens (Spain). Sensors were prepared by grinding composite powder 1 $\supset$ PEDOT(45) (ca. $30 \mathrm{mg}$ ) in ethanol $(300 \mu \mathrm{L})$ to obtain a homogeneous suspension, then drop-casting $8 \mu \mathrm{L}$ of this suspension onto the interdigitated electrode and letting the sensor dry at room temperature. Sensors exhibited a resistance of ca. $20 \mathrm{k} \Omega$. This value is in the ideal range of our setup ( $100 \Omega$ to $1 \mathrm{M} \Omega$ ) for an accurate chemiresistive detection as it gives a high signal/noise ratio. This composite was selected as it was the lowest loaded in the adequate conductivity range, and thus the most porous for a high gas accessibility.

Sensors were then introduced in the sensing chambers and the resistance was monitored using a Keithley 4200-SCS analyzer, under a measuring bias of 0.1 V. Samples were degassed under reduced pressure (1 Pa) until stabilization of the electrical resistance. The chamber was then purged several times with $\mathrm{N}_{2}$ (Tomoe Shokai co., Japan) until no resistance change (attributed to grain displacement under pressure) could be observed under an inert gas atmosphere. Sensing experiments were performed by introducing a mixture of $\mathrm{NO}_{2}$ in $\mathrm{N}_{2}$ (certified at $98.7 \mathrm{ppm}$, Tomoe Shokai co., Japan) at the desired pressure inside the sensing chamber.

\begin{tabular}{|c|c|c|c|c|c|}
\hline $\begin{array}{c}\text { EDOT } \\
\text { loading }(\boldsymbol{\%})^{\mathbf{a}}\end{array}$ & $\begin{array}{c}\text { S/Cr } \\
(\mathbf{a t .})\end{array}$ & $\begin{array}{c}\text { Polymerization } \\
\text { yield }(\boldsymbol{\%})^{\mathbf{b}}\end{array}$ & $\begin{array}{c}\text { PEDOT mass } \\
\text { fraction }(\boldsymbol{\%})^{\mathbf{c}}\end{array}$ & I/S (at.) & $\begin{array}{c}\text { (PEDOT + I) } \\
\text { fraction in the } \\
\text { composite (\%) }\end{array}$ \\
\hline 14 & 0.19 & 84 & 10 & 2.00 & 23 \\
\hline 25 & 0.36 & 85 & 18 & 1.43 & 33 \\
\hline 45 & 0.63 & 84 & 27 & 0.61 & 36 \\
\hline 77 & 1.07 & 83 & 39 & 0.74 & 51 \\
\hline 101 & 1.40 & 82 & 45 & 0.60 & 55 \\
\hline 153 & 2.22 & 86 & 57 & 0.53 & 66 \\
\hline
\end{tabular}

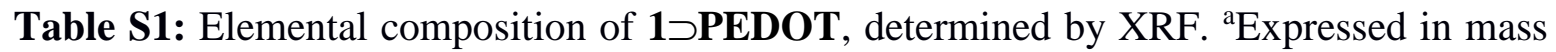
empty of 1 host. ${ }^{b}$ Defined as the atomic ratio of $S$ in the composite after washing, divided by the amount of $\mathrm{S}$ introduced. ${ }^{\mathrm{c}} \mathrm{Calculated}$ from the $\mathrm{S} / \mathrm{Cr}$ ratio for a system comprising pristine PEDOT and 1. This value is independent of the doping state, and was used for the designation of the samples. The actual mass fraction of PEDOT in the system varies depending on the mass of the additional dopant. 


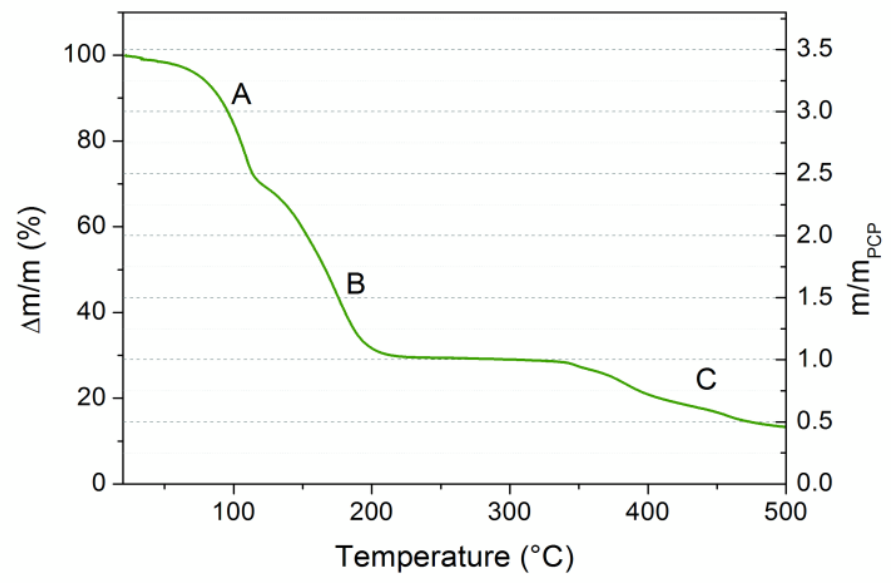

Figure S1: Determination of the maximum loading of EDOT in $\mathbf{1}$ by thermogravimetric analysis. The right-hand axis corresponds to the mass of compound normalized by the mass of 1. A slight excess of EDOT was introduced in 1 using the diethyl ether evacuation technique. The TGA curve presents three decrease steps: (A) Evaporation of weakly bound EDOT (outside 1); (B) Evacuation of the strongly bound EDOT (adsorbed in 1); (C) Decomposition of $\mathbf{1}$.

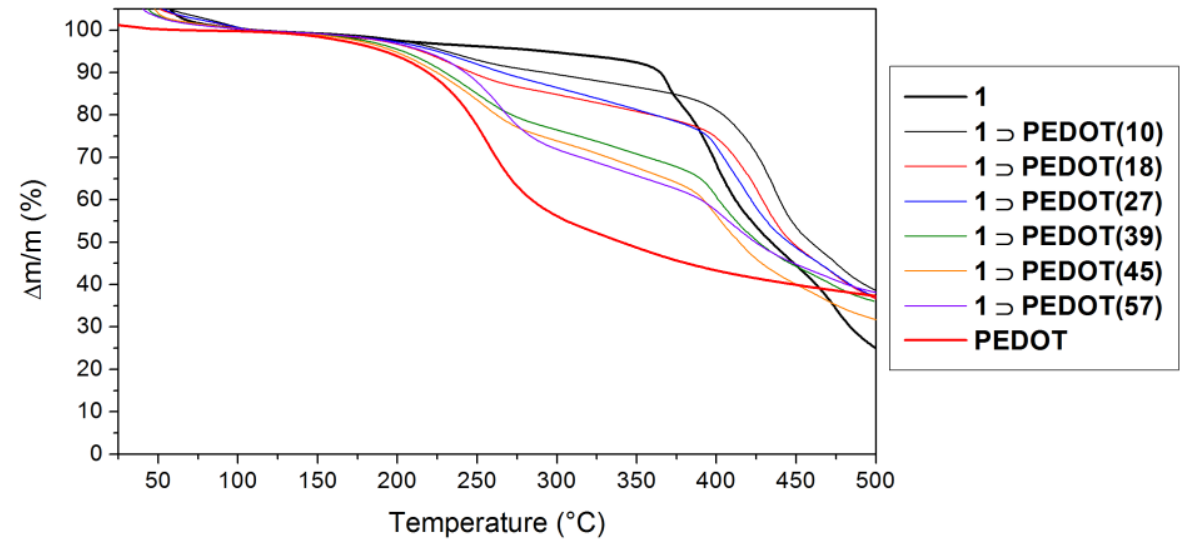

Figure S2: Thermogravimetric analysis of 1 $\supset$ PEDOT composites. Measurements were performed under a $\mathrm{N}_{2}$ flux, with a heating rate of $5{ }^{\circ} \mathrm{C} / \mathrm{min}$. For clarity, the curves have been renormalized so that $100 \%$ corresponds to the composites after evacuation of adsorbed water. As PEDOT does not decompose fully before the decomposition of $\mathbf{1}$, the determination of the polymer content presents some incertitude. In addition, interaction between PEDOT and 
the PCP could potentially modify the decomposition kinetics and behavior of the system (e.g. formation of chromium sulfide instead of chromium oxide). However, under the assumption of an identical mass loss for doped PEDOT in all composites, the mass loss obtained by TGA are similar to the ones obtained for (PEDOT + I) calculated by XRF (cf Table S1).
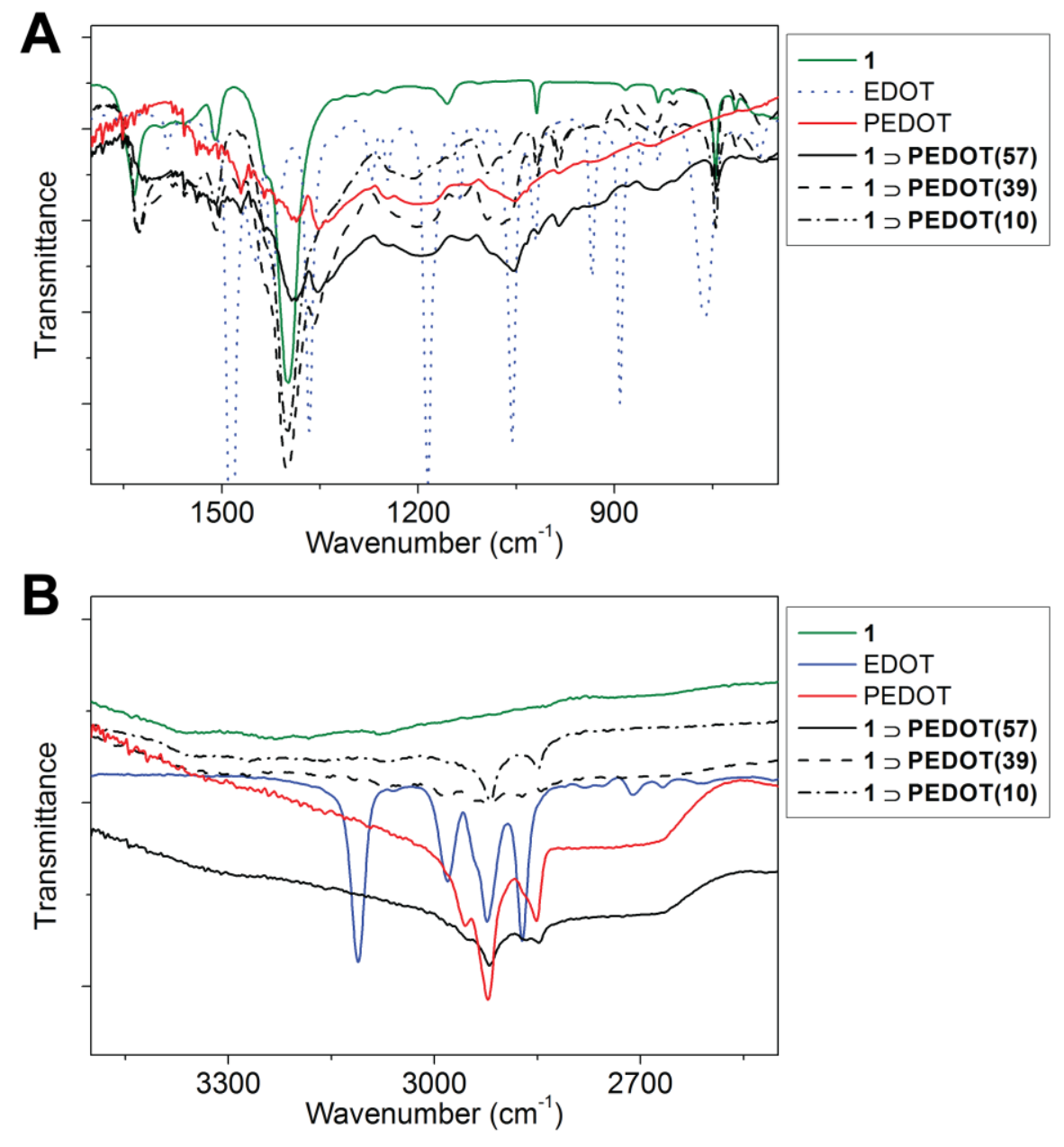

Figure S3: ATR-IR spectra of several 1つPEDOT composites. Spectra of host PCP 1, EDOT monomer, and PEDOT are also represented for reference. The presence of $\mathbf{1}$ can be identified by the presence of specific peaks that don't overlap with peaks of PEDOT. Principal peaks are present at $1625 \mathrm{~cm}^{-1}, 1508 \mathrm{~cm}^{-1}, 1017 \mathrm{~cm}^{-1}$ and $750 \mathrm{~cm}^{-1}$, in agreement with the literature. ${ }^{3}$ Similarly, presence of oxidized PEDOT can be attested to by the presence of peaks at $840 \mathrm{~cm}^{-1}, 980 \mathrm{~cm}^{-1}, 1190 \mathrm{~cm}^{-1}$, and $1350 \mathrm{~cm}^{-1}$, as identified by the literature. ${ }^{4}$ 


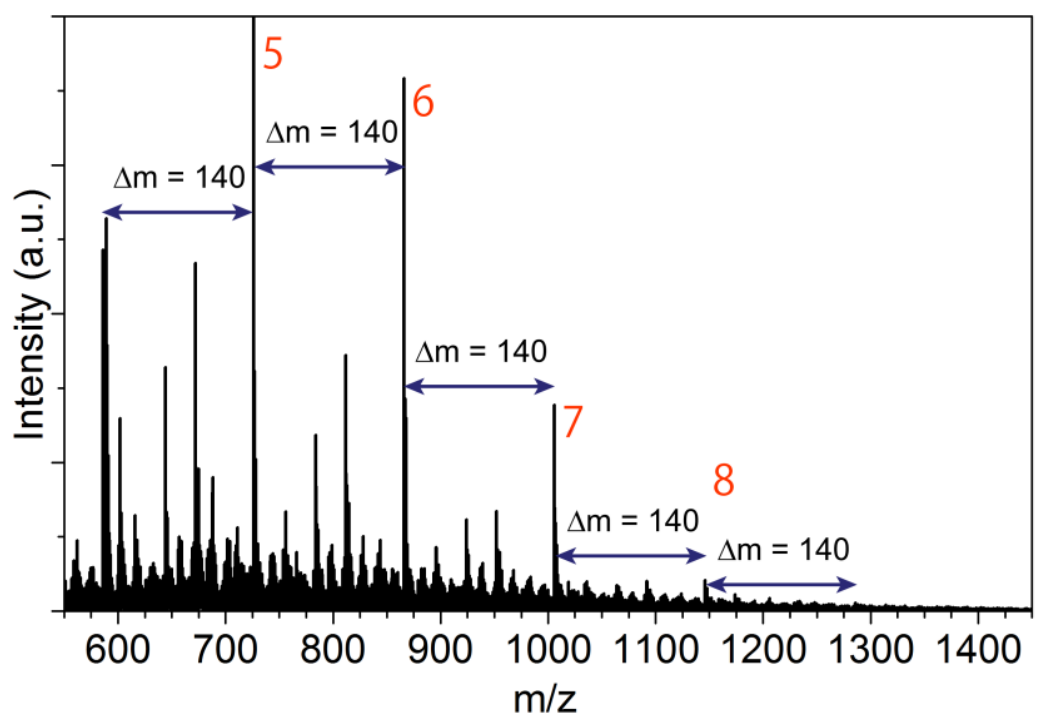

Figure S4: MALDI-TOF spectrum of PEDOT extracted from 1 $\supset$ PEDOT. Ionization was performed using a terthiophene matrix. The peaks correspond to sodium-adducts of PEDOT. The numbers in red correspond to the polymerization degree of the associated peak. Note that ionization may vary greatly with the PEDOT chain length and that the MALDI-TOF spectrum may not represent accurately the chain length distribution. More specifically, the average molecular weight $\mathrm{M}$ of the sample may be underestimated as low molecular weight species can be more easily detected.

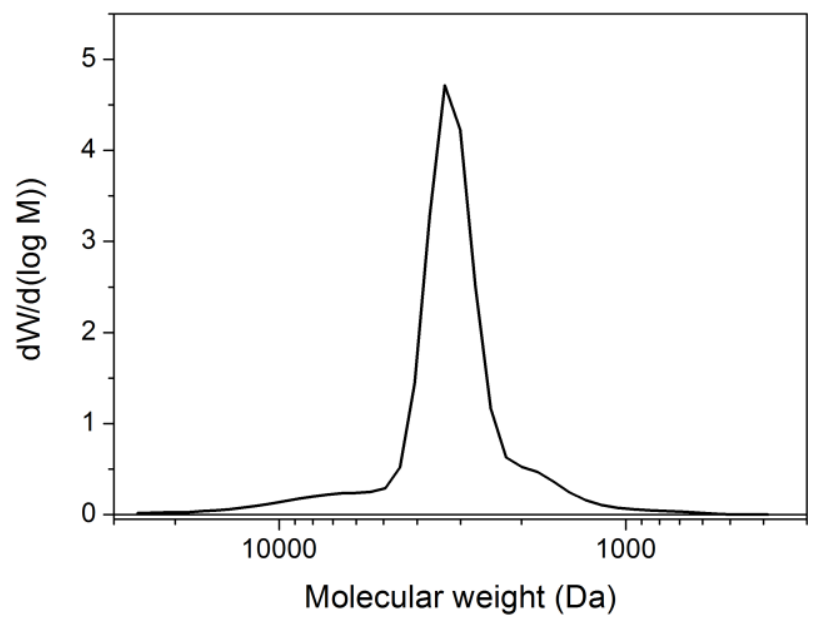

Figure S5: Molecular weight distribution of PEDOT extracted from 1 $\supset$ PEDOT, obtained by Gel Permeation Chromatography $\left(\mathrm{GPC}, M_{\mathrm{n}}=2960, M_{\mathrm{w}}=3560 \mathrm{Da}\right.$, PDI $\left.=1.2\right)$. The 
molecular weights were determined using polystyrene standards. Note that GPC molecular weight determination is affected by the hydrodynamic radius and thus by the rigidity of the polymer chain. As PEDOT possess a conjugated back-bone, it is more rigid than polystyrene (which is itself considered as a rigid polymer). As a consequence, $\mathrm{M}$ values obtained by GPC may be overestimated. Considering the values obtained by GPC and MALDI-TOF, we can speculate that the PEDOT is present in the composite as chains of intermediate molecular weights.

A
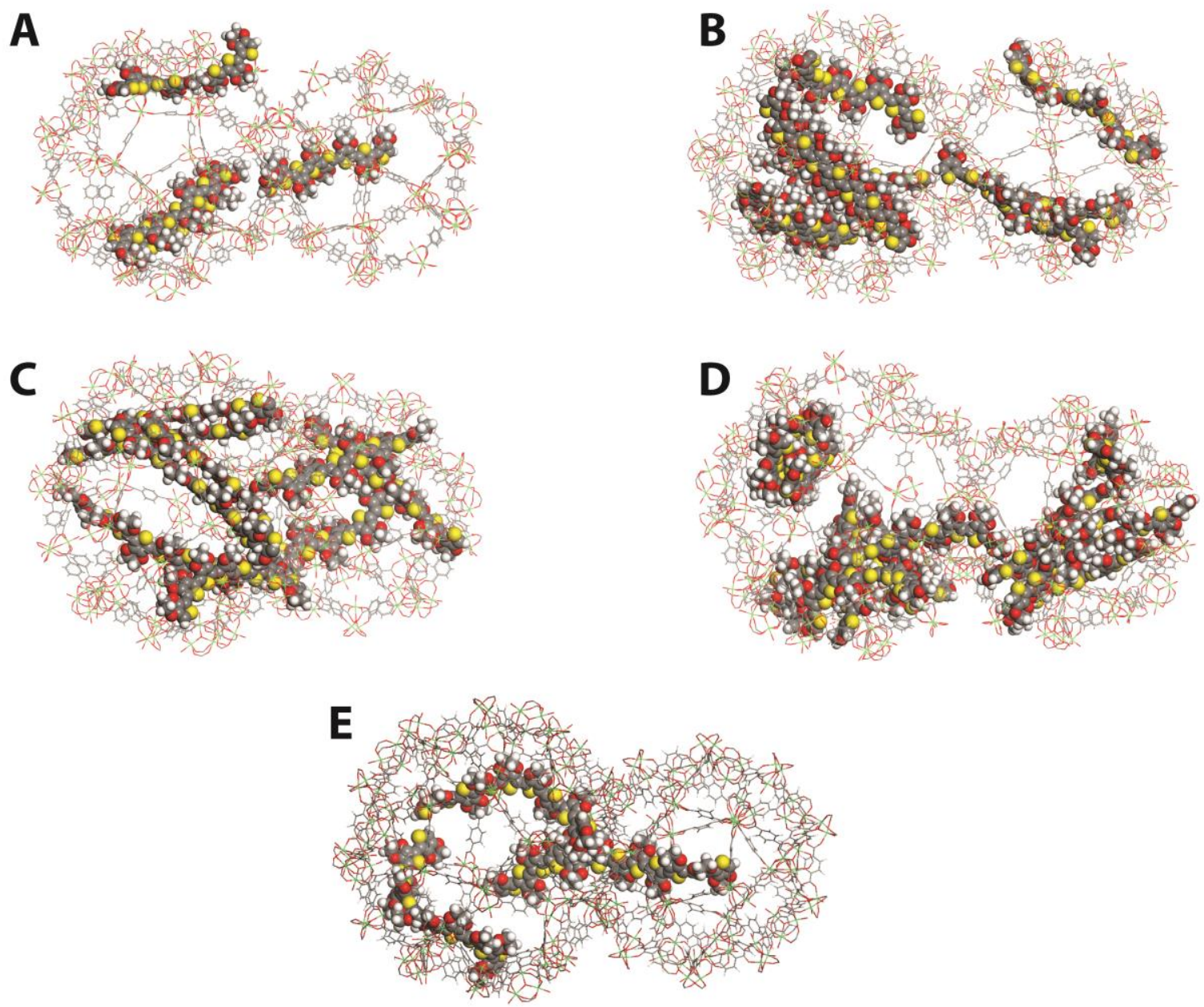

Figure S6: Molecular dynamics (MD) structures of PEDOT chains in two adjacent

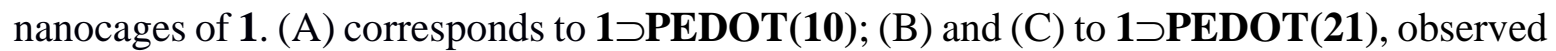
along two different angles, (D) to $1 \supset$ PEDOT(29) and (E) to $1 \supset$ PEDOT(13). PEDOT chains were introduced in the PCP as octamers in (A) to (D), and as dodecamers in (E). We can 
observe that for the low loading rates, PEDOT chains interact with the cages walls and leave the inside of the pores empty. For higher loading rates, the PEDOT chains also interact between each other to form loosely packed clusters. The remaining porosity inside the cages is then less well-defined.

Gray: carbon; White: hydrogen; Red: oxygen; Yellow: sulfur; Green: chromium. MD simulations were performed using the Material Studio Modeling v8.0 software package (Accelrys Inc, San Diego, CA, USA), using the Universal Force Field, as implemented in the Forcite module. The initial structure was based on the X-ray crystal structure of $\mathbf{1}$. The quench dynamics with the optimized structures were conducted at $473 \mathrm{~K}$, and then, MD calculations were carried out at $298 \mathrm{~K}$ for 200 ps under NVE conditions.

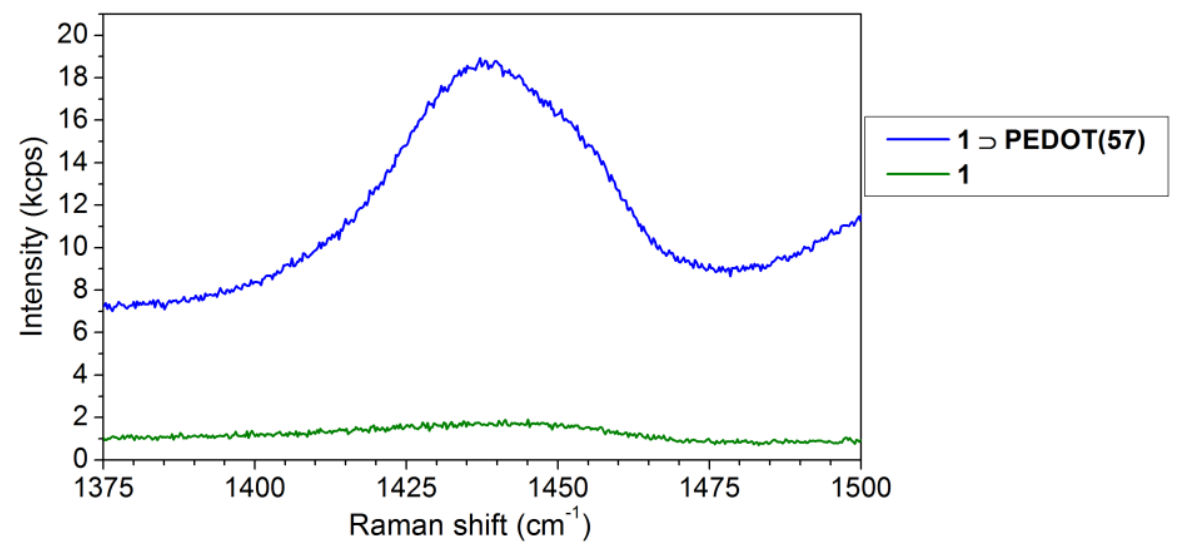

Figure S7: Raman spectra of 1 and 1つPEDOT(57) (excitation wavelength: $532 \mathrm{~nm}$ ). 1 $\supset$ PEDOT (57) shows the $\mathrm{C}_{\alpha}=\mathrm{C}_{\beta}$ symmetric stretching mode at $1440 \mathrm{~cm}^{-1}$, indicating that the dominant resonance form of PEDOT chains in $\mathbf{1}$ is the benzoid form. ${ }^{5}$ The benzoid form reveals a coiled conformation, whereas the quinoid form is indicative of a linear conformation. ${ }^{6}$ Thus, Raman spectroscopy suggested that PEDOT inside $\mathbf{1}$ forms a coiled structure. This is consistent with the results of MD simulation, in which the PEDOT chains have to coil to adopt the inner curvature of the cages of $\mathbf{1}$. 


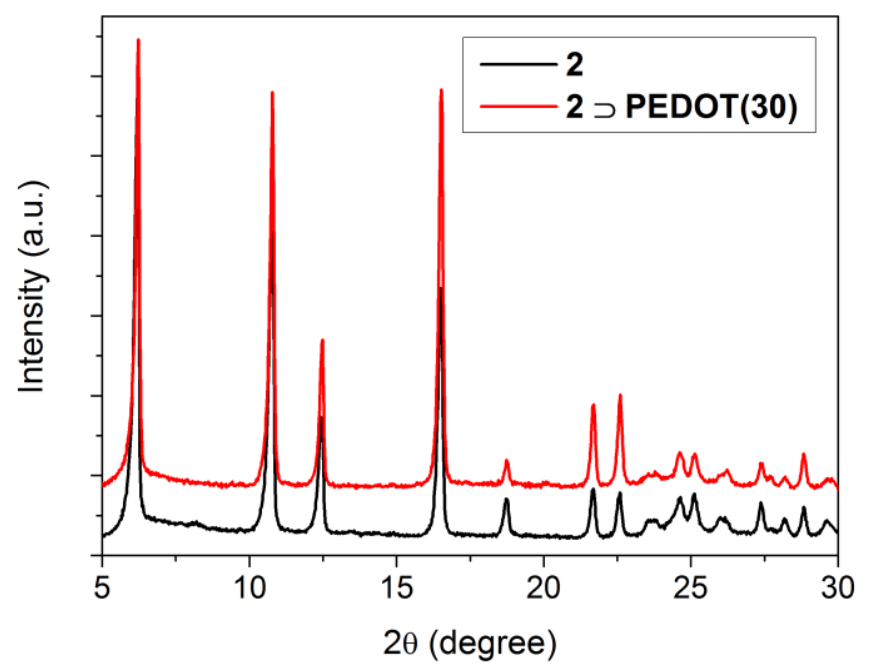

Figure S8: X-Ray Diffractograms of 2 and $2 \supset$ PEDOT.

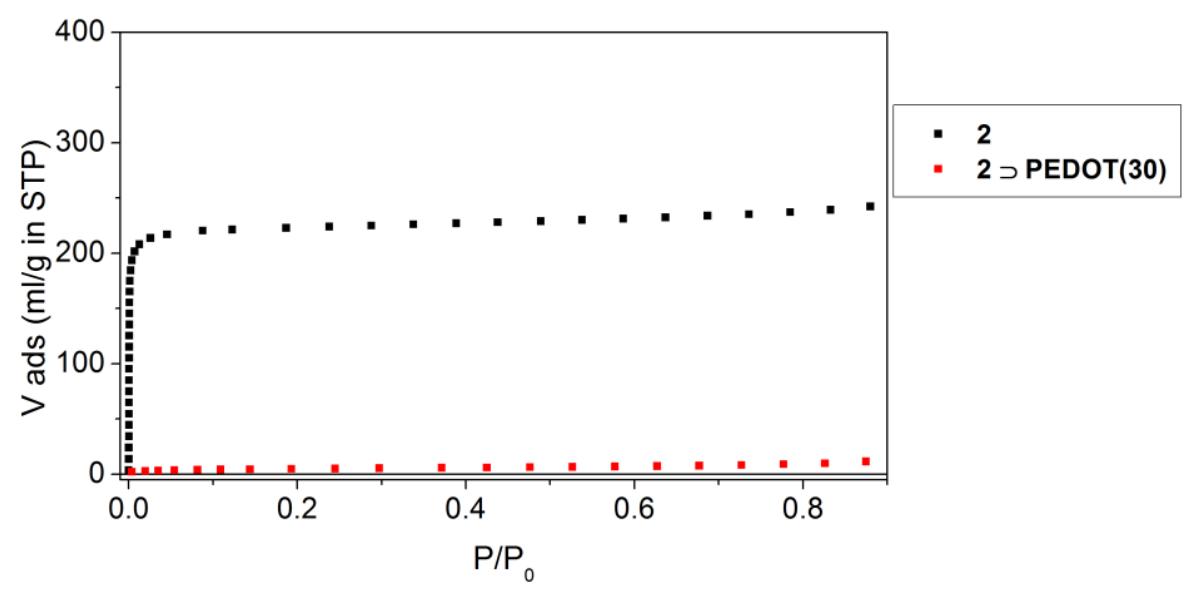

Figure S9: $\mathrm{N}_{2}$ adsorption isotherms $(77 \mathrm{~K}$ ) of 2 and $2 \supset$ PEDOT. 

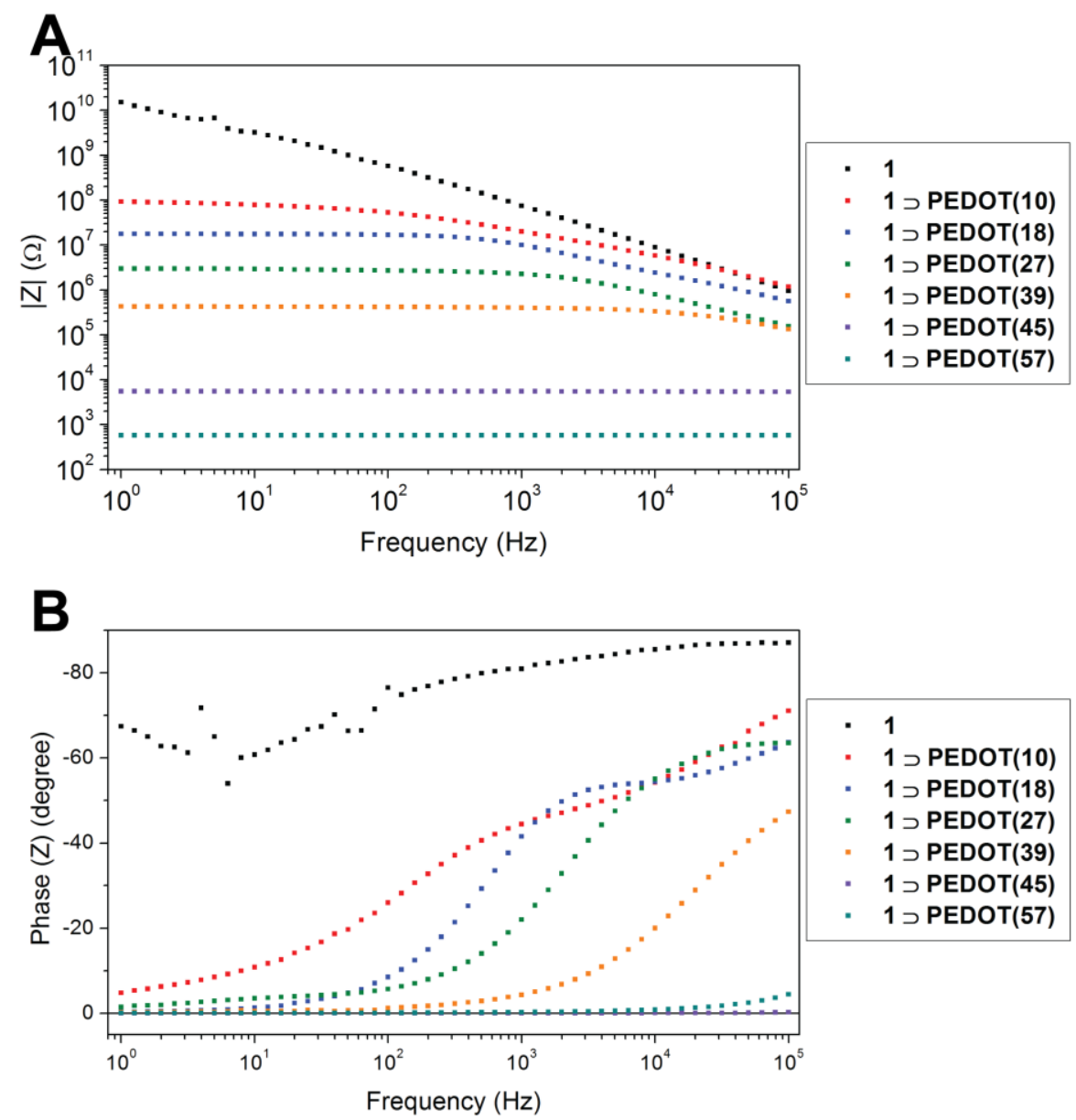

Figure S10: Impedance diagrams of $1 \supset$ PEDOT (Bode representation: (A) Modulus and (B) Phase).

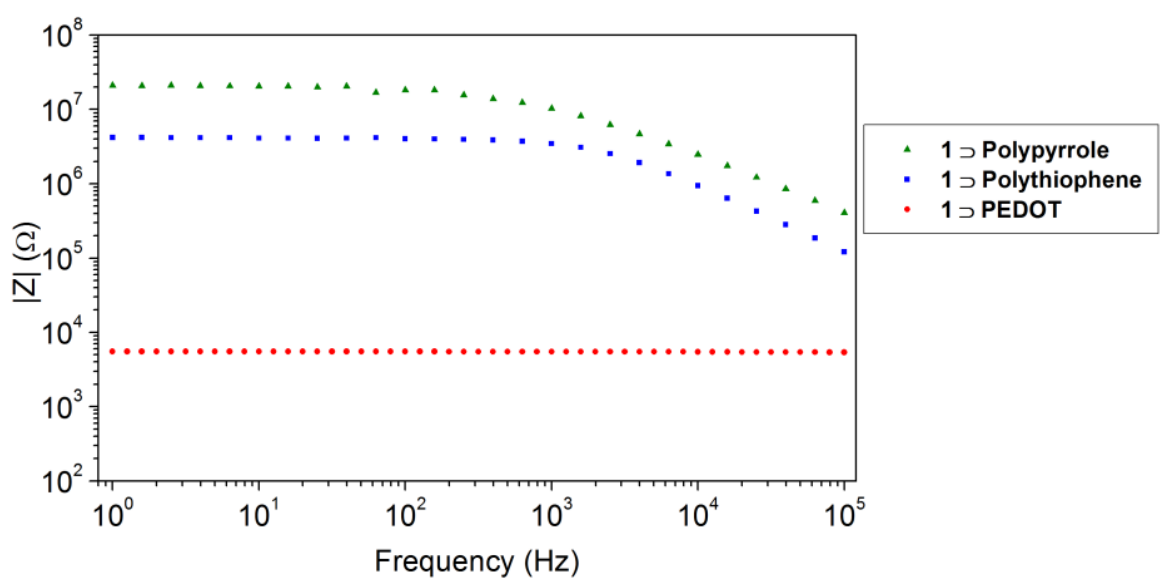

Figure S11: Impedance diagrams of composites of 1 with several polymers (polypyrrole, polythiophene and PEDOT). Samples were prepared by introducing the same amount of 


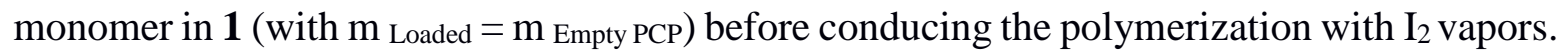
For PEDOT, the sample corresponds to $1 \supset$ PEDOT(45).

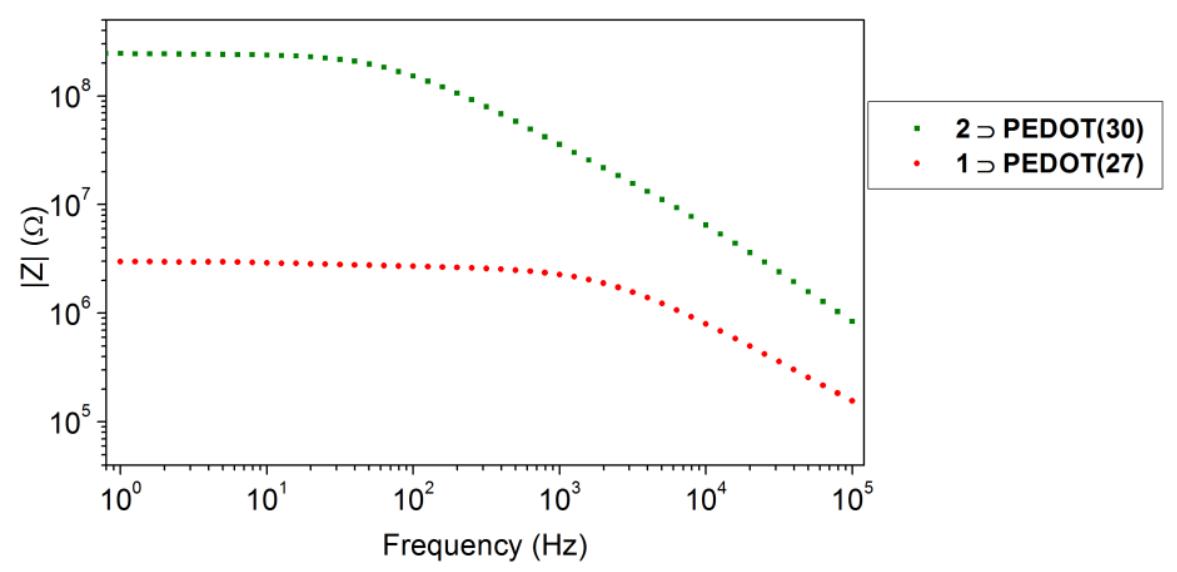

Figure S12: Impedance diagrams of composites of 2 $\supset$ PEDOT(30). 1 $\supset$ PEDOT(27) is added for comparison.

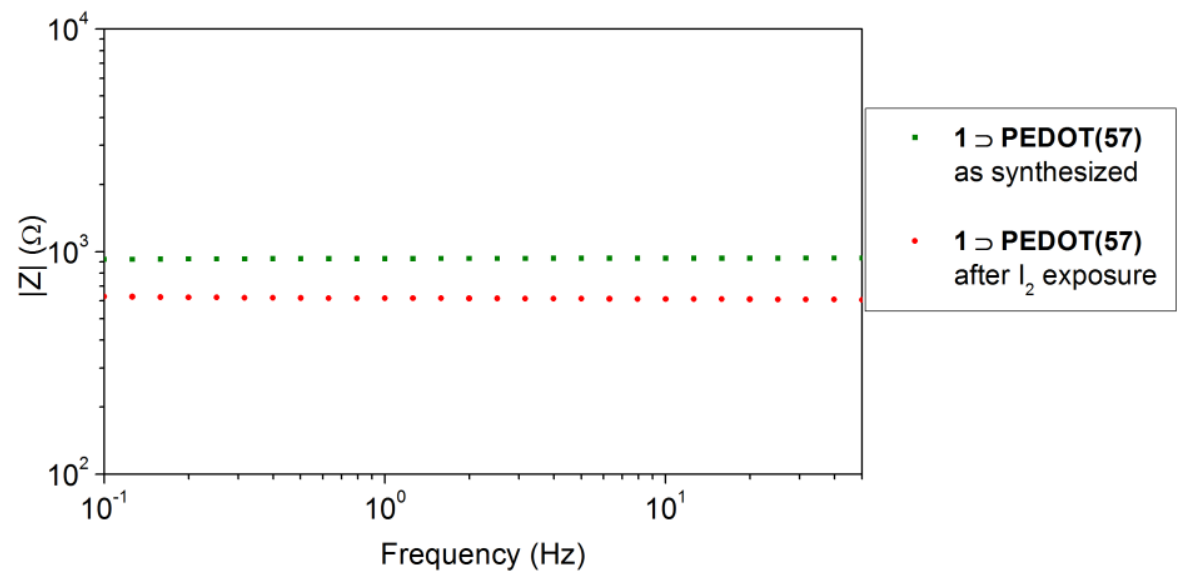

Figure S13: Impedance diagrams of composites of 1つPEDOT(57) as synthesized (i.e. natively doped with non-volatile iodine species), and after exposure to iodine vapors. The conductivity was increased only by a factor 1.7. Furthermore, the re-doped samples released volatile $I_{2}$ over time, and could not be used for gas sensing. For these reasons, we preferred to work only with natively doped samples, as these materials didn't release any volatile species after evacuation at $150{ }^{\circ} \mathrm{C}$ under vacuum. 


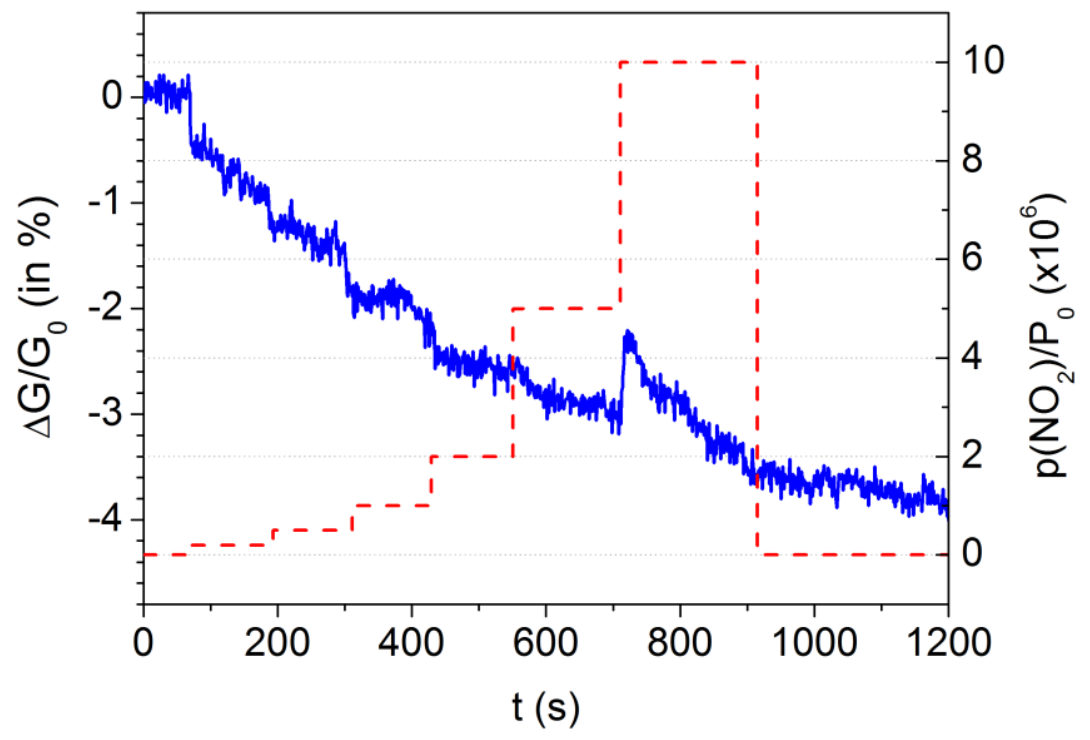

Figure S14: Transient conductivity measurement of a sensor prepared using PEDOT obtained from solution polymerization in Toluene, instead of $1 \supset$ PEDOT.

\section{References}

(1) Férey, G.; Mellot-Draznieks, C.; Serre, C.; Millange, F.; Dutour, J.; Surblé, S.; Margiolaki, I. Science 2005, 309, 2040.

(2) Devic, T.; Serre, C.; Audebrand, N.; Marrot, J.; Férey, G. J. Am. Chem. Soc. 2005, $127,12788$.

(3) Liu, Q.; Ning, L.; Zheng, S.; Tao, M.; Shi, Y.; He, Y. Sci. Rep. 2013, 3, 2916.

(4) Zhang, J.; Zhao, X. S. J. Phys. Chem. C 2012, 116, 5420.

(5) Garreau, S.; Louarn, G.; Buisson, J. P.; Froyer, G.; Lefrant, S. Macromolecules 1999, 32, 6807.

(6) Tang, F.-C.; Chang, J.; Wu, F.-C.; Cheng, H.-L.; Hsu, S. L.-C.; Chen, J.-S.; Chou, W.Y. J. Mater. Chem. 2012, 22, 22409. 